\title{
Why Interstellar Ice Dust Grains Should Be Elongated
}

\author{
P. M. Bellan (iD) \\ Applied Physics and Materials Science, California Institute of Technology, Pasadena, CA 91125, USA; pbellan@caltech.edu \\ Received 2020 August 25; revised 2020 October 12; accepted 2020 October 26; published 2020 December 17
}

\begin{abstract}
Models of interstellar dust alignment assume that dust grains are elongated, but none of these models explain why dust grains should be elongated. On the other hand, models of interstellar dust grain growth assume that dust grains are spherical and not elongated. We show that when dusty plasma effects and the dipole moment of water molecules are together taken into account, ice grains in interstellar space should be prolate ellipsoids and not spheres. Dusty plasma analysis shows that an ice grain is charged to a negative potential that has magnitude nearly equal to that of the electron temperature. Several different mechanisms causing deviation from sphericity are identified; these mechanisms involve the interaction of the dipole moment of water molecules with electric fields associated with ice grain charging. These mechanisms include the focusing of water molecule trajectories, the migration of water molecules in a quasi-liquid layer on the grain surface toward regions where the electric field is strongest, the enhancement of this migration by the bombardment of energetic protons that gain energy upon falling into the ice grain negative potential, and mutual repulsion by electric charges having the same sign. The aspect ratio is established shortly after the ice grain is formed, and then is maintained as the grain grows.
\end{abstract}

Unified Astronomy Thesaurus concepts: Very small grains (1770); Interstellar dust processes (838); Laboratory astrophysics (2004); Interstellar magnetic fields (845); Interstellar molecules (849); Interstellar emissions (840); Starlight polarization (1571); Water vapor (1791); Plasma astrophysics (1261); Ice formation (2092)

\section{Introduction}

The classic model of interstellar dust by Mathis et al. (1977; MRN) suggests that dust is spherical and has a size distribution scaling as $r^{-3.5}$ over the range $0.005-0.25 \mu \mathrm{m}$. This model is very widely used but requires a small radius cutoff to avoid divergence of the mass, which would scale as $\int r^{3} r^{-3.5} d r$. While MRN acknowledged that dust might not be spherical, virtually all users of the MRN model assume spherical dust grains.

The alignment of interstellar dust to the local magnetic field, first observed by Hiltner (1949) and Hall (1949), provides a valuable diagnostic for the interstellar magnetic field. However, in remarkable contrast to the MRN model assumption that dust grains are spherical, the interpretation of dust magnetic alignment requires the dust to be ellipsoidal as sketched in Figure 2 of Lazarian (2007).

Various models for dust magnetic field alignment exist. The original models of dust alignment assume that ellipsoidal dust grains develop a magnetic moment either from being electrically charged and rotating (Martin 1971) or from having intrinsic paramagnetism (Davis \& Greenstein 1951) or ferromagnetism (Jones \& Spitzer 1967). The magnetic moment, however produced, is then assumed to precess with respect to the magnetic field. A second set of models assumes that plasma winds develop as a result of magnetohydrodynamic forces and that these winds tend to align the dust grains (Gold 1952). The third and most recent set of models assumes that torques produced by radiation pressure cause dust grains to spin and then align (Lazarian 2007). The word "alignment" requires that the dust grains be nonspherical. Thus, while the various alignment models differ, a common feature is that dust grains are assumed to be ellipsoidal and prolate.

A critical question then is why interstellar dust grains should be prolate. This question is not answered by standard models of dust growth as these models typically assume that dust is spherical (e.g., see Acharyya et al. 2011). We present here semiquantitative arguments showing that consideration of dusty plasma physics concepts, dipole dynamics, and ellipsoid electrostatics shows that astrophysical dust grains should spontaneously develop a prolate shape. The model will be presented in the context of the formation of water ice dust grains since this is what has been observed in an astrophysically relevant laboratory experiment, but it should also apply to other situations where molecules having a substantial electric dipole moment are accreted. While the model is rough, it outlines the main effects that need to be considered, and presents initial estimates of their relative importance.

\section{Observations and Properties that Provide a Basis for the Model}

Before presenting the model we summarize several observations and properties that provide a basis for it.

Observations that electric fields have a strong effect on water ice formation. Bartlett et al. (1963) showed via a laboratory experiment operating at atmospheric pressure that the growth of ice crystals is greatly accelerated in the presence of an electric field exceeding $5 \times 10^{4} \mathrm{~V} \mathrm{~m}^{-1}$. Although the precise mechanism was not determined, evidence pointed to the importance of accreted water molecules easily migrating on the surface of the ice crystals as shown by Mason et al. (1963). Laboratory measurements by Libbrecht \& Tanusheva (1999) showed that at atmospheric pressure, an electric field accelerates ice crystal growth by a factor of 5-50, and they attributed this to the electric force on a dipole $\sim-\nabla(\boldsymbol{p} \cdot \boldsymbol{E})$ overcoming surface tension. Here $\boldsymbol{p}$ is the water molecule dipole moment and $\boldsymbol{E}$ is an imposed electric field.

Rapid migration of water molecules in a thin quasi-liquid layer (QLL) on ice surfaces. Murphy (1953) examined the migration of water molecules on the surface of ice crystals and showed that this could be considered as a bipedal walk where the two hydrogen atoms of a water molecule constitute the biped legs and the energy required for detaching each leg from the surface is $0.23 \mathrm{eV}$. The orientation of the water dipole is 
such that the hydrogen atoms are positive and the oxygen atom is negative so the legs of the biped are positive and thus stick to a negatively charged surface. This migration provides a microscopic correspondence to atomic force microscope measurements by Doppenschmidt \& Butt (2000), indicating that ice is coated by a liquid water film having a thickness of a few nanometers. Mason et al. (1963) noted that adsorbed water molecules migrate for considerable distances over a crystal surface and may travel from one crystal face to another before evaporating or becoming built into the crystal. Thus, an ice dust grain can be considered to be coated with a thin (about nanometer-scale) layer of fluid-like molecules that can easily move over the surface and so seek the location with the lowest potential energy. This layer is known as a quasi-liquid layer (QLL).

Laboratory experiments demonstrating rapid formation of water molecules in a weakly ionized deuterium-oxygen plasma and then rapid formation of ice grains. Shimizu et al. (2010) created a deuterium-oxygen weakly ionized plasma and observed rapid growth of water vapor. When cooled electrodes were used, water ice grains spontaneously formed, giving an ice dusty plasma.

Laboratory experiments demonstrating spontaneous formation and rapid growth of prolate ice grains. Prolate ice dust grains were observed to form spontaneously in a series of laboratory experiments at Caltech in a cold weakly ionized plasma with injected water vapor (Chai \& Bellan 2013, 2015a, 2015b; Marshall et al. 2017). The maximum size and maximum prolate shape occurred at the lowest operating pressures and for the lowest-mass background gas species. The elongated dust grains sometimes had a lattice-like arrangement with the long axes of the dust grains parallel to each other. The lattice-like structure is typical of previous dusty plasmas where spherical plastic grains were used. The parallel alignment of the elongated dust grains in the Caltech experiments was explained by the charge on the prolate dust grains being concentrated at the ends so that the lowest-energy lattice would have aligned grains. These experiments also indicate that the dipole moment of water molecules is critical for ice formation because ice was formed with two other molecules having dipole moments (methanol, acetone) but ice was not formed when a molecule with no dipole moment was used (carbon dioxide). This shows that the existence of a molecular dipole moment must be central to any model of ice formation in a weakly ionized plasma. The fractional ionization and neutral gas temperature in these experiments were similar to those in the interstellar medium, but the gas density was many orders of magnitude higher.

Repulsion of two charges. The general tendency of a structure with total charge $Q$ to become elongated can be seen by considering two identical conducting spheres of radius $R$ with centers separated by a distance $\gg R$ and connected by a thin wire. Because the two spheres are electrically connected, they are at the same potential $V$. The charge on sphere \#1 is denoted $q_{1}=\beta Q$ and the charge on sphere \#2 is denoted $q_{2}=(1-\beta) Q$, where $0 \leqslant \beta \leqslant 1$. The capacitance of each sphere is $C=4 \pi \varepsilon_{0} R$, so the total electrostatic energy is $q_{1}^{2} / 2 C+q_{2}^{2} / 2 C=\left(\beta^{2}+(1-\beta)^{2}\right) Q^{2} / 2 C$. This energy minimizes when $\beta=1 / 2$, so the lowest energy state is for half the charge to be on each sphere. Since the two half-charges mutually repel, there is a force directed toward increasing the separation between spheres. Thus, a highly elongated charged prolate ellipsoid will have half the charge at each of the two sharp ends, and these will repel each other, creating a force that tends to make the ellipsoid more prolate. This behavior is demonstrated in a more formal way by noting that the electrostatic energy $Q^{2} / 2 C$ of a conducting ellipsoid with fixed charge $Q$ decreases when the ellipsoid becomes prolatethat is, the capacitance $C$ increases (Shchukin \& Grigorev 1998; Holgate \& Coppins 2016).

\section{Model}

The dust grain grows around an initial core. This core might form by the spontaneous nucleation of water vapor as was observed in the Caltech laboratory experiments, or the core could be a speck of refractory material as is typically assumed for astrophysical situations. Spontaneous formation would require charging of an initial cluster of water molecules by the free electrons in the weakly ionized plasma (homogeneous nucleation). In the interstellar situation, which contains atoms, molecules, and specks of solid, the core could be a non-ice material such as silicate or carbon (heterogeneous nucleation). Interstellar space is assumed to be a weakly ionized, mainly hydrogen plasma with a number density $n=10^{7} \mathrm{~m}^{-3}$, a gas temperature $T_{\mathrm{g}} \simeq 10^{-2} \mathrm{eV}=100 \mathrm{~K}$, an electron temperature $T_{\mathrm{e}} \simeq 1 \mathrm{eV}$, and an ice temperature $T_{\mathrm{i}}=10-100 \mathrm{~K}$.

There are several possible mechanisms for why the presence of weakly ionized plasma should cause the dust grains to become prolate spheroids. The relative importance of these mechanisms will depend on the size of the ice grain so one mechanism might dominate when the ice grain is formed and then another might dominate as the ice grain grows beyond a critical size. A mechanism that can be immediately rejected is isotropic accretion since this would eventually cause any initial shape to become spherical.

Before presenting the model, we first summarize certain relevant geometric and electrical properties of conducting ellipsoids and certain relevant properties of orbital motionlimited (OML) theory, a theory that describes the effective fluxes of charged particles impacting a dust grain. Because the initial dust core is in a weakly ionized plasma, it will be impacted by both electrons and ions and so will become charged. This charging is described by OML theory (Spitzer 1941; Allen 1992; Barkan et al. 1994; Shukla \& Mamun 2002). This theory takes into account the angular momentum of incident charged particles to provide a focusing effect for attractive electrostatic forces and a Boltzmann-like attenuation for repulsive forces. It also shows that because the electron thermal velocity greatly exceeds the ion thermal velocity, a dust grain charges up to a negative voltage that is about $T_{\mathrm{e}}$. This analysis is independent of the fractional ionization and so dust grains charge up to this negative voltage even if the fractional ionization is extremely low. For example, laboratory plasma dust grains charge up to this negative voltage when the ionization fraction is $\sim 10^{-6}$. The electrons constituting this charge move freely on the surface during the timescales of interest so the grain surface is an equipotential (Bartlett et al. 1963). The ice grain can thus be considered to be a charged conductor. Since the Debye length and collision mean free paths are orders of magnitude larger than the grain dimensions, the motion of particles in the vicinity of the grain can be considered as collisionless trajectories in vacuum with the trajectories being governed by orbital mechanics.

Summary of geometric and electrical properties of ellipsoids. The electrical properties of a conducting ellipsoid are 
well known. Using cylindrical geometry the surface of an ellipsoid is prescribed by

$$
\frac{r^{2}}{b^{2}}+\frac{z^{2}}{a^{2}}=1
$$

The ellipsoid volume is $4 \pi a b^{2} / 3$ and the surface area is (Zwillinger 2011)

$$
S=2 \pi b^{2}+2 \pi \frac{a b}{\sqrt{1-b^{2} / a^{2}}} \sin ^{-1} \sqrt{1-b^{2} / a^{2}} .
$$

The aspect ratio is defined as $\alpha=a / b$, so a prolate ellipsoid has $\alpha>1$. It is useful to compare various ellipsoid properties to the corresponding properties of a sphere having the same volume. On defining $R$ to be the radius of a reference sphere having the same volume as the ellipsoid, i.e., $4 \pi a b^{2} / 3=4 \pi R^{3} / 3$, it is seen that

$$
\begin{gathered}
a=\alpha^{2 / 3} R \\
b=\alpha^{-1 / 3} R .
\end{gathered}
$$

The surface area of an ellipsoid normalized to that of a sphere having the same volume is thus

$$
\frac{S}{4 \pi R^{2}}=\frac{1}{2} \alpha^{-2 / 3}+\frac{1}{2} \frac{\alpha^{1 / 3}}{\sqrt{1-1 / \alpha^{2}}} \sin ^{-1} \sqrt{1-1 / \alpha^{2}}
$$

this normalized surface has a minimum at $\alpha=1$.

The surface charge density of a conducting ellipsoid having total charge $Q$ is

$$
\sigma(r, z)=\frac{Q}{4 \pi b^{2} a \sqrt{\frac{r^{2}}{b^{4}}+\frac{z^{2}}{a^{4}}}} .
$$

Using Equation (1) to solve for $r(z)$, the surface charge density can be expressed in terms of a location $z$ on the surface as

$$
\sigma(z)=\frac{Q}{4 \pi b a \sqrt{1-\frac{z^{2}}{a^{2}}\left(1-\frac{1}{\alpha^{2}}\right)}}
$$

which shows that $\sigma(a)=\alpha \sigma(0)$. The electric field normal to the surface of the ellipsoid is $E_{\mathrm{n}}=\sigma / \varepsilon_{0}$, so $E_{\mathrm{n}}(a)=\alpha E_{\mathrm{n}}(0)$. Thus, the normal electric field on the surface at the end $z=a$ of a prolate ellipsoid is $\alpha$ times larger than the normal electric field on the surface at the side $z=0$.

The potential $V$ on the surface of the conducting ellipsoid is

$$
V=\frac{Q}{8 \pi \varepsilon_{0} a \sqrt{1-1 / \alpha^{2}}} \ln \left(\frac{1+\sqrt{1-1 / \alpha^{2}}}{1-\sqrt{1-1 / \alpha^{2}}}\right)
$$

so the capacitance is

$$
C=\frac{8 \pi \varepsilon_{0} \alpha^{2 / 3} R \sqrt{1-1 / \alpha^{2}}}{\ln \left(\frac{1+\sqrt{1-1 / \alpha^{2}}}{1-\sqrt{1-1 / \alpha^{2}}}\right)}
$$

Since a sphere of radius $R$ has a capacitance $C_{\text {ref }}=4 \pi \varepsilon_{0} R$, the capacitance of an ellipsoid normalized to the capacitance of a same-volume sphere is

$$
\frac{C(\alpha)}{C_{\mathrm{ref}}}=\frac{2 \alpha^{2 / 3} \sqrt{1-1 / \alpha^{2}}}{\ln \left(\frac{1+\sqrt{1-1 / \alpha^{2}}}{1-\sqrt{1-1 / \alpha^{2}}}\right)} ;
$$

this shows that the normalized capacitance is at a minimum when $\alpha=1$.

On combining Equations (7)-(9) and using $E_{\mathrm{n}}=\sigma / \varepsilon_{0}$ the normal electric field at a location $z$ on the surface of the ellipsoid is

$$
E_{\mathrm{n}}(z)=\frac{1}{\sqrt{1-\frac{z^{2}}{a^{2}}\left(1-\frac{1}{\alpha^{2}}\right)}} \frac{2 \alpha^{1 / 3} \sqrt{1-1 / \alpha^{2}}}{\ln \left(\frac{1+\sqrt{1-1 / \alpha^{2}}}{1-\sqrt{1-1 / \alpha^{2}}}\right)} \frac{V}{R} .
$$

Lack of significant OML focusing of water molecules. It is assumed that the interstellar medium contains water molecules with an abundance that is a small fraction of the hydrogen abundance. Because of collisions that occur between water molecules and hydrogen molecules at locations very far from the ice grain, the water molecules are assumed to be in thermal equilibrium with the hydrogen gas, so both water and hydrogen molecules have the same temperature $T_{\mathrm{g}}$. Now consider a water molecule traveling toward the dust grain with an initial velocity $v_{\mathrm{i}}=\sqrt{2 \kappa T_{\mathrm{g}} / m_{\mathrm{w}}}$, where $m_{\mathrm{w}}$ is the water molecule mass. The water molecule has an intrinsic electric dipole moment $p=6.17 \times 10^{-30} \mathrm{C} \mathrm{m}$. The water molecule motion is governed by an extension of OML theory that takes into account forces on the electric dipole moment. The impact parameter for a grazing collision is $h$, so any molecule with an impact parameter less than $h$ collides with the dust grain. The situation of a water molecule with an initial velocity perpendicular to the ellipsoid $z$ axis will first be considered; this is sketched in Figure 1. Such a water molecule has an orbital angular momentum $L=m_{\mathrm{w}} h v_{\mathrm{T}}$ with respect to the $z$-axis. The water molecule is assumed to be oriented such that $-\nabla(\boldsymbol{p} \cdot \boldsymbol{E})$ produces a force $-p \nabla|E|$ toward the ice grain. Thus, the water molecule is attracted to the ice grain much like an iron filing is attracted to a magnet (Chai \& Bellan 2015b). According to OML theory the molecule will follow a curved trajectory that conserves both $L$ and energy. Upon colliding with the dust grain the molecule has electrostatic potential energy $-p E_{\mathrm{n}}$. Conservation of energy gives

$$
\frac{1}{2} m_{\mathrm{w}} v_{\mathrm{i}}^{2}=\frac{1}{2} m_{\mathrm{w}} v_{\mathrm{f}}^{2}-p E_{\mathrm{n}}(z)
$$

where $v_{\mathrm{f}}$ is the velocity of the grazing collision. Since $L$ is conserved, $h v_{\mathrm{i}}=b v_{\mathrm{f}}$, so Equation (12) can be expressed as

$$
h^{2}=(1+F(z)) b^{2}
$$

where using $\kappa T_{\mathrm{g}} \approx m_{\mathrm{w}} v_{\mathrm{i}}^{2} / 2$, Equation (11), and $V \approx T_{\mathrm{e}}$ the focusing enhancement factor $F$ is

$$
\begin{aligned}
F(z)= & \frac{p E_{\mathrm{n}}(z)}{\kappa T_{\mathrm{g}}}=\frac{1}{\sqrt{1-\frac{z^{2}}{\alpha^{2} b^{2}}\left(\alpha^{2}-1\right)}} \\
& \times \frac{2 \alpha^{1 / 3} \sqrt{1-1 / \alpha^{2}}}{\ln \left(\frac{1+\sqrt{1-1 / \alpha^{2}}}{1-\sqrt{1-1 / \alpha^{2}}}\right)} \frac{p}{\kappa R} \frac{T_{\mathrm{e}}}{T_{\mathrm{g}}} .
\end{aligned}
$$

At $z=0$ the focusing enhancement is $F(0) \approx T_{\mathrm{e}} p / R \kappa T_{\mathrm{g}}$ for $1 \leqslant \alpha<5$, and at $z=a$, the focusing enhancement is $F(a)=\alpha F(0)$. 


\section{(a) End view}

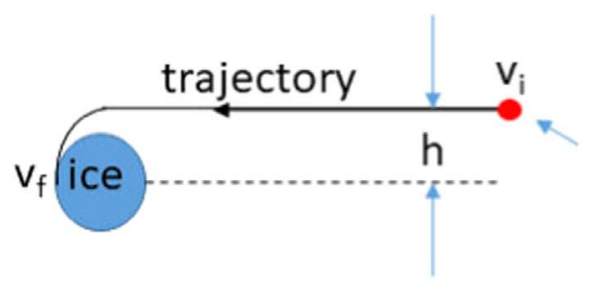

(b) Top view

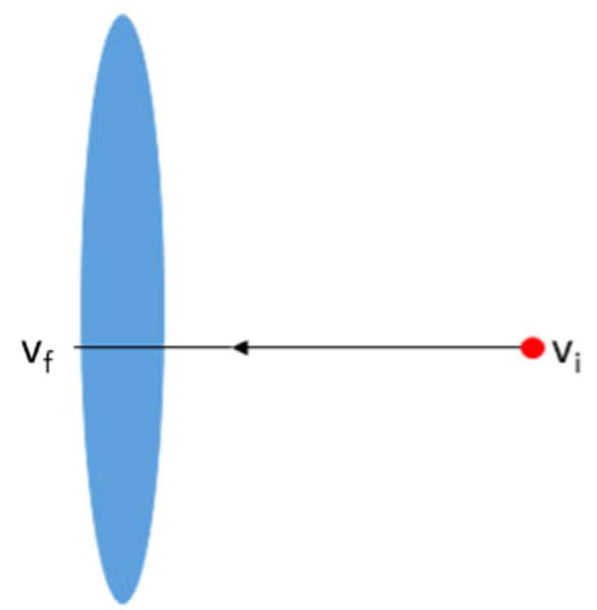

Figure 1. (a) View along $z$-axis of water molecule colliding with prolate ice grain. (b) Top view.

The ellipsoid has a projected area $\pi a b$ and with focusing equation (13) shows that the cross section becomes $\pi a b(1+$ $F(z))^{1 / 2}$, which is analogous to the focusing of particles attracted to a cylindrical Langmuir probe (Allen 1992). Focusing is significant only if $T_{\mathrm{e}} p / R \kappa T_{\mathrm{g}}>1$, i.e., if $R<p T_{\mathrm{e}} / \kappa T_{\mathrm{g}}=4 \mathrm{~nm}$, for $T_{\mathrm{e}} / T_{\mathrm{g}}=10^{2}$ At larger $R$ (e.g., at micrometer-scale) OML focusing is insignificant, so while OML focusing might be important when the ice dust grain first forms, OML focusing can be ignored when the grain becomes substantially larger. This lack of focusing means that only incident water molecules directly targeting the ice grain will hit the ice grain.

Enhanced accretion at ends of prolate ellipsoid. Although ellipsoids with dimensions substantially exceeding $4 \mathrm{~nm}$ have no OML focusing, there is a geometrical effect that causes enhanced accretion at the ends ( $z= \pm a$ vicinity). We consider the number of molecules that make nearly normal impact with the ice surface as representative of all molecules that impact the surface at the same point, and construct rays that emanate from the ice surface to infinity with the density of rays at the surface proportional to the local electric field on the surface. Since the water molecule mean free path is much larger than the ice grain dimensions, water molecules coming from infinity that make near normal impact with the ice grain will travel inward toward the ice grain along these rays. At large distances from the water molecule, the rays are indistinguishable, so each ray will carry the same number of water molecules as any other ray. Since Equation (11) shows that $E_{\mathrm{n}}(a) / E_{\mathrm{n}}(0)=\alpha$, the flux of water molecules impacting the surface of the ice grain at $z= \pm a$ will be $\alpha$ times the flux impacting the surface at $z=0$. This means that

$$
\dot{a}_{\mathrm{accr}}=\alpha \dot{b}_{\mathrm{accr}}
$$

where $\dot{a}_{\text {accr }}$ and $\dot{b}_{\text {accr }}$ are the respective rates of increase of $a$ and $b$ as a result of the accretion of water molecules coming from infinity. This means that accretion of water molecules incident from infinity will sustain but not increase $\alpha$. This effect can be understood by noting that if the end regions are approximated as hemispheres while the mid-region is approximated as a finite-length cylinder, then the normal rays to each hemisphere subtend nearly $2 \pi$ steradians, while normal rays to the finitelength cylinder subtend the much smaller balance of the total $4 \pi$ steradians available. Each normal ray impacting a point on the surface is of course accompanied by non-normal rays that provide a flux scaling of $\hat{n} \cdot \hat{s}$, where $\hat{n}$ is the normal to the surface and $\hat{s}$ is the ray direction. Defining $\hat{n} \cdot \hat{s}=\cos \theta$, averaging over all rays corresponds to averaging $\cos \theta$ for $-\pi /$ $2<\theta<\pi 2$, which gives a factor of $2 / \pi$ that will be ignored in view of the uncertainties in other parameters.

If the ellipsoid is replaced by an equal-volume sphere of radius $R$, then conservation of flux shows that $\rho_{\mathrm{i}} \dot{R}=n_{\mathrm{w}} m_{\mathrm{w}} v_{\mathrm{w}}$, where $\rho_{\mathrm{i}}$ is the ice density, $n_{\mathrm{w}}$ is the density of gas-phase water molecules, $m_{\mathrm{w}}$ is the mass of a water molecule, and $v_{\mathrm{w}}$ is the thermal velocity of gas-phase water molecules. Since accretion is proportional to the incoming flux, which is proportional to the surface electric field, it is seen that $\dot{b}_{\text {accr }} / \dot{R}=E_{\mathrm{n}}(0) R / V$ since $V / R$ is the electric field of the equal-volume sphere. Thus

$$
\begin{aligned}
\dot{b}_{\mathrm{accr}} & =E_{\mathrm{n}}(0) \frac{R}{V} \frac{n_{\mathrm{w}} m_{\mathrm{w}}}{\rho_{\mathrm{i}}} v_{\mathrm{w}} \\
& =\frac{2 \alpha^{1 / 3} \sqrt{1-1 / \alpha^{2}}}{\ln \left(\frac{1+\sqrt{1-1 / \alpha^{2}}}{1-\sqrt{1-1 / \alpha^{2}}}\right)} \frac{n_{\mathrm{w}} m_{\mathrm{w}}}{\rho_{\mathrm{i}}} v_{\mathrm{w}}
\end{aligned}
$$

and

$$
\dot{a}_{\mathrm{accr}}=\frac{2 \alpha^{4 / 3} \sqrt{1-1 / \alpha^{2}}}{\ln \left(\frac{1+\sqrt{1-1 / \alpha^{2}}}{1-\sqrt{1-1 / \alpha^{2}}}\right)} \frac{n_{\mathrm{w}} m_{\mathrm{w}}}{\rho_{\mathrm{i}}} v_{\mathrm{w}} .
$$

Inadequacy of migration of water molecules on surface as a result of molecule thermal random motion. An incident molecule that impacts the ice grain does not immediately become part of the ice crystal structure but instead becomes a member of the QLL coating the ice. A water molecule in the QLL on the ice surface feels a force $\nabla\left(p E_{\mathrm{n}}\right)=p \nabla E_{\mathrm{n}}$ toward the region of strongest electric field, and if the mobility is $\mu$, then the water molecules constituting the QLL will have, as sketched in Figure 2, a drift in the tangential direction

$$
v_{\tan }=p \mu \hat{t} \cdot \nabla E_{\mathrm{n}},
$$

where $\hat{t}$ is a unit vector tangent to the surface.

Using the Einstein relation $D=\mu \kappa T_{\mathrm{i}}$, where $D$ is the surface diffusivity and $T_{\mathrm{i}}$ is the ice temperature, the tangential velocity of the molecules constituting the QLL will be

$$
v_{\tan }=\frac{p D}{\kappa T_{\mathrm{i}}} \hat{t} \cdot \nabla E_{\mathrm{n}} .
$$




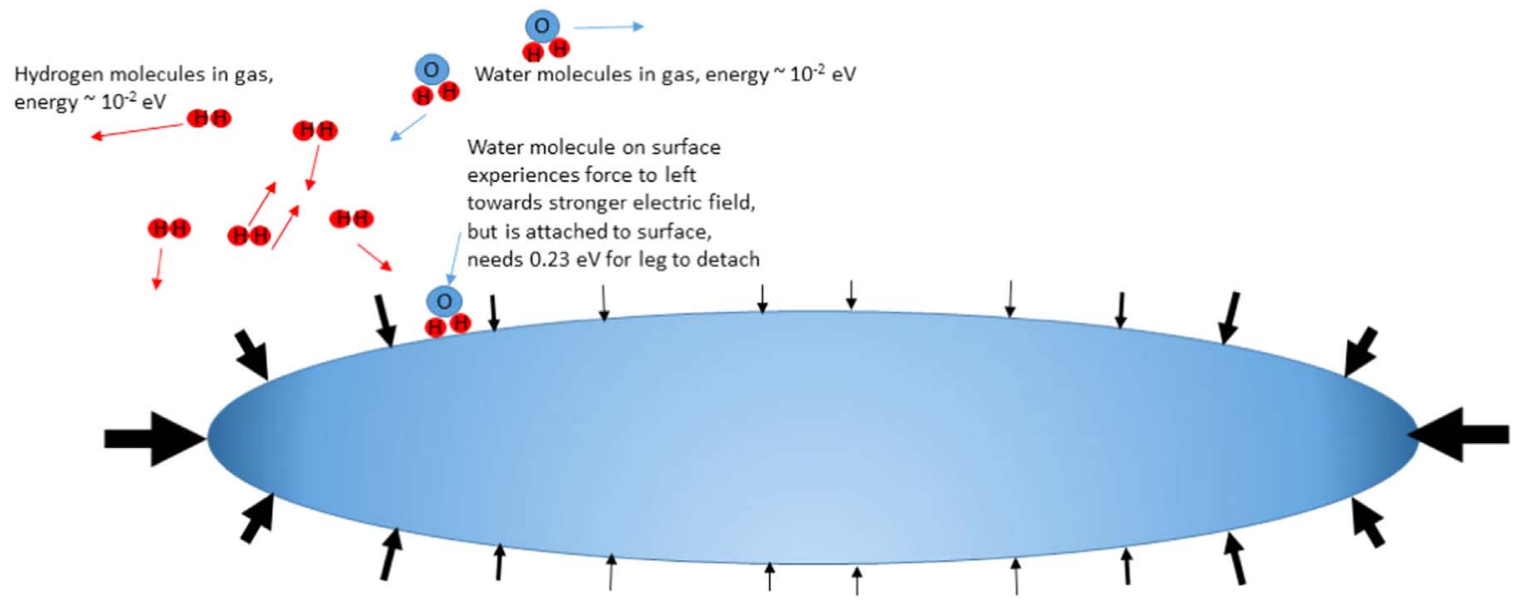

Black arrows denote normal electric field on surface Wider, bigger arrows denote stronger field

Figure 2. Water molecule in the QLL on the ice ellipsoid surface experiences a force along the surface pushing the water molecule toward a stronger normal electric field, i.e., toward an end. Here, only neutral gas particles are shown (hydrogen molecules, water molecules) and these have energies of $\sim 10^{-2} \mathrm{eV}$ corresponding to $\sim 100 \mathrm{~K}$.

Since $E_{\mathrm{n}}(a)$ at the tip is $\alpha$ times $E_{\mathrm{n}}(0)$ at the side,

$$
\begin{aligned}
\hat{t} \cdot \nabla E_{\mathrm{n}} & \approx \frac{E_{\mathrm{n}}(a)-E_{\mathrm{n}}(0)}{a} \\
& =\frac{\alpha-1}{a} E_{\mathrm{n}}(0) \\
& \approx \frac{\alpha-1}{a} \frac{|V|}{b}
\end{aligned}
$$

SO

$$
v_{\tan } \approx p \frac{D}{\kappa T_{\mathrm{i}}} \frac{\alpha-1}{a} \frac{|V|}{b}
$$

and the time for a molecule to travel from $z=0$ to $z=a$ will be

$$
t=\frac{\alpha}{\alpha-1} \frac{\kappa T_{\mathrm{i}} R^{3}}{p D|V|} .
$$

The molecular diffusion of water molecules in the QLL on ice has been measured down to $237 \mathrm{~K}$ by Price et al. (1999) and modeled to $230 \mathrm{~K}$ using a molecular dynamics simulation by Gladich et al. (2011), who obtained slightly lower values than Price et al. but following a similar dependence. The Gladich et al. result can be expressed as

$$
\begin{aligned}
D & =1.5 \times 10^{2} \exp \left(-\frac{6900}{T_{\text {ice }, \mathrm{K}}}\right) \mathrm{m}^{2} \mathrm{~s}^{-1} \\
& =1.5 \times 10^{2} \exp \left(-\frac{0.59}{T_{\text {ice }, \mathrm{eV}}}\right) \mathrm{m}^{2} \mathrm{~s}^{-1}
\end{aligned}
$$

where $T_{\text {ice, } \mathrm{K}}$ is the ice temperature in kelvin and $T_{\mathrm{ice}, \mathrm{eV}}$ is the ice temperature in electronvolts.

Suppose $Z \gg 1$, where $Z$ is the number of electrons on a grain, the initial grain is nearly spherical, and from OML charging theory the grain surface potential is $V \simeq T_{\mathrm{e}}$, i.e., about $1 \mathrm{~V}$ (negative). Since the grain surface potential is given by

$$
V=\frac{Z q_{\mathrm{e}}}{4 \pi \varepsilon_{0} R}
$$

these assumptions imply

$$
R \gg \frac{q_{\mathrm{e}}}{4 \pi \varepsilon_{0} T_{\mathrm{e}}}=1.4 \mathrm{~nm}
$$

Thus, we assume an initial radius $R=10 \mathrm{~nm}$ corresponding to $Z=7$.

Marshall \& Bellan (2020) measured the gas temperature in the Caltech ice dusty plasma experiment to be $190 \mathrm{~K}$ using laser-induced fluorescence; inserting $190 \mathrm{~K}$ in Equation (23) gives $D=2.5 \times 10^{-14} \mathrm{~m}^{2} \mathrm{~s}^{-1}$. Using this diffusion coefficient, the time for a surface molecule to move from $z=0$ to $z=a$ is

$$
t=10^{-2} \frac{\alpha}{\alpha-1}\left(\frac{R}{10 \mathrm{~nm}}\right)^{3} \mathrm{~s} .
$$

As the grain becomes more spherical (i.e., $\alpha \rightarrow 1$ ), the elongation time increases and becomes infinite for a grain that is a perfect sphere. If $T=100 \mathrm{~K}$ is assumed, the diffusivity given by Equation (23) and hence the elongation time increase by a factor of $\exp \left(-6900\left(\frac{1}{190}-\frac{1}{100}\right)\right) \sim 10^{14}$, so the time would become of the order of $5 \times 10^{4} \mathrm{yr}$. Thus, the mobility of thermal QLL molecules is marginally adequate for $10 \mathrm{~nm}$ grains in the Caltech experiment and may be adequate for astrophysical situations but becomes insufficient if the grain size substantially exceeds $10 \mathrm{~nm}$.

Enhanced mobility via proton bombardment. Murphy (1953) suggested that a surface water molecule attached to ice can be thought of as a biped (e.g., a human-like two-footed creature). Each foot is one of the two hydrogen atoms constituting the molecule and the thorax or main body is the oxygen atom. The hydrogen atoms are regions of positive charge and the oxygen atom is negative-hence the dipole moment. The energy required to detach one foot from the ice surface is the hydrogen bond energy, which is $0.23 \mathrm{eV}$, and the energy required to detach both feet is double this value. Murphy postulated that if an energy $W$ was applied to the molecule with $0.23 \mathrm{eV}<W<0.46 \mathrm{eV}$, one foot would detach and the molecule would make a step of about $0.8 \AA$ A. These steps would be random and so would constitute the 


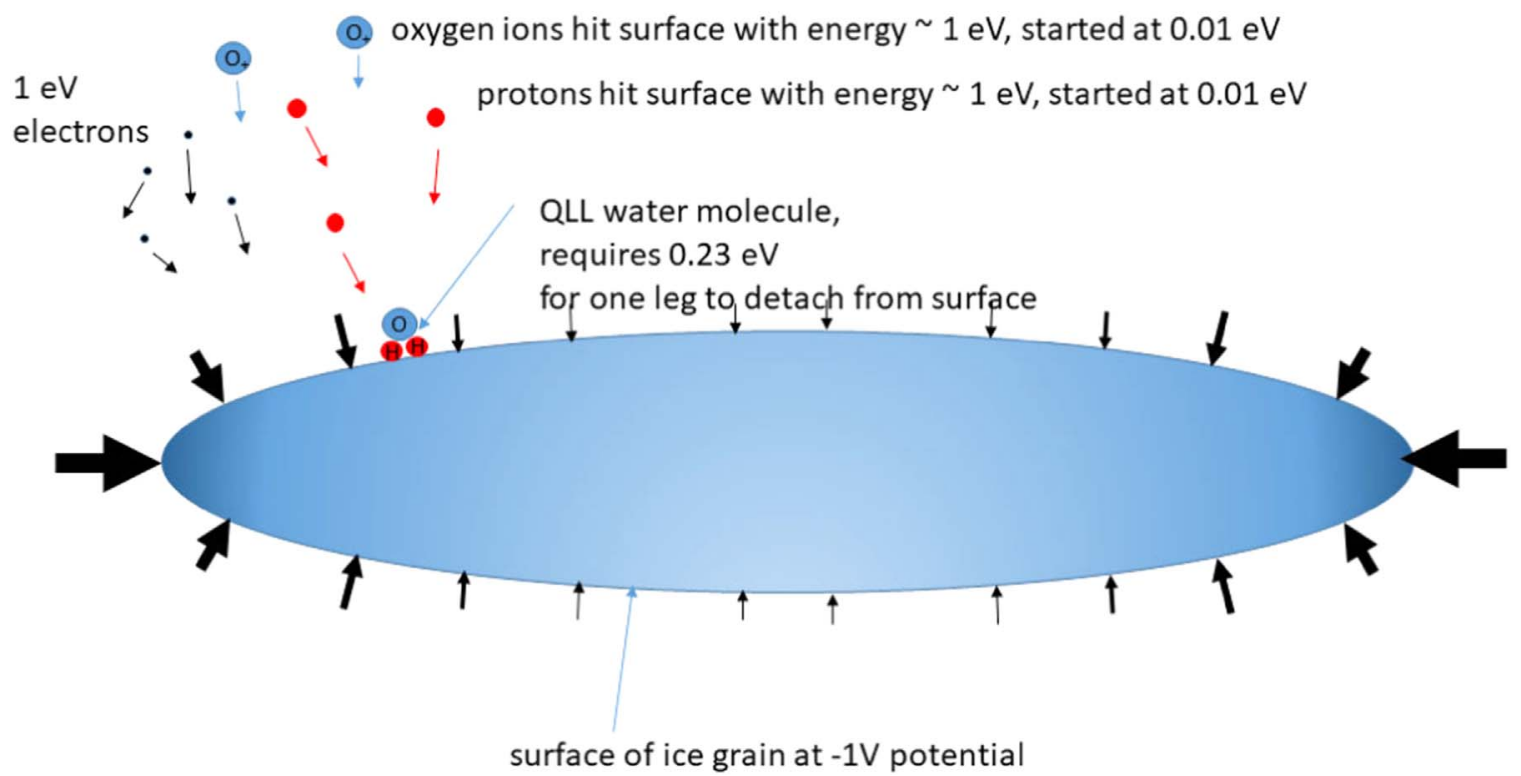

Figure 3. Same as Figure 2, except now gas-phase charged particles are shown and gas-phase neutral particles are not shown. Upon colliding with the ice grain, the protons have a kinetic energy corresponding to the negative potential on the ice grain.

random steps in a diffusion process that would then map to a mobility via the Einstein relation $D=\mu \kappa T$, where $T$ is the temperature of the molecules involved. Since only molecules with energy exceeding $0.23 \mathrm{eV}$ could make steps, the diffusion process would contain an Arrhenius coefficient $\exp \left(-U_{0} / \kappa T\right)$, where $U_{0}$ would exceed $0.23 \mathrm{eV}$. This is roughly in line with diffusion coefficients containing an $\exp \left(-U_{0} / \kappa T\right)$ factor; different models have different values for $U_{0}$ but generally have $U_{0}$ in the range of $0.2-0.6 \mathrm{eV}$.

However, the Murphy model, the Gladich et al. model, and similar models do not consider the situation where the ice is immersed in a weakly ionized plasma. We now show that the existence of surrounding plasma changes the situation completely because in a plasma the ice is negatively charged and so is bombarded by ions that gain kinetic energy as they fall in the negative potential of the ice grain. Because ice grains are charged to a negative voltage that is approximately the electron temperature, the kinetic energy of these infalling ions is thus given by the electron temperature, not by the ice temperature nor by the temperature of the exterior neutral gas. The ion bombardment energy thus has no dependence on the very cold temperatures in the system, that is, temperatures in the 10-200 K range. The OML model of dust charging shows that because of the enhancement of the effective cross section for ions, the ion flux at the dust grain surface is increased by a factor $\left|\mathrm{eV} / \kappa T_{\mathrm{i}}\right| \approx T_{\mathrm{e}} / T_{\mathrm{i}}$ relative to the ion thermal flux at infinity. Since the ions in a weakly ionized hydrogen plasma are protons, an ice grain in a weakly ionized plasma is bombarded by a flux $\sim n_{\mathrm{p}} v_{\mathrm{g}} T_{\mathrm{e}} / T_{\mathrm{i}}$ of protons with $1 \mathrm{eV}$ kinetic energy, where $n_{\mathrm{p}}$ is the proton density at a large distance and $v_{\mathrm{g}}$ is the thermal velocity of the incoming protons when they are far from the ice grain and not yet significantly accelerated. This proton bombardment is sketched in Figure 3.

For a head-on elastic collision of a particle $m_{1}$ with a stationary target particle $m_{2}$, the fraction of incident particle kinetic energy transferred to the target particle is $4\left(1+m_{1} / m_{2}\right)^{-2} m_{1} / m_{2}$, while a grazing collision will involve a smaller energy transfer. Because a water molecule is 18 times as heavy as a proton, upon making an elastic collision with a water molecule a proton transfers as much as $20 \%$ of its kinetic energy to the water molecule in each collision. Thus, if the electron temperature exceeds about $1.3 \mathrm{eV}$, an incident proton will transfer more than $0.23 \mathrm{eV}$ to a water molecule attached to the surface and so detach one foot of the water molecule, which can then make a random step of size $0.8 \AA$. Heavier ions would transfer a greater fraction of their energy during an elastic collision and so would be more likely to detach the water molecule from the surface. The proton might also knock the water molecule off completely, but not with so much energy that it flies off to infinity so it might come back to some distant location on the ice surface. This idea that protons give gentle kicks that stimulate migration but heavier ions would knock molecules off completely is consistent with the observation shown in Figure 2 of Chai \& Bellan (2015a) that ice dust grains become smaller when heavier background gases are used. An incident proton that transfers some fraction of its energy to a surface water molecule in the QLL would become trapped in the negative potential well of the ice grain and so after the initial collision would make a number of subsequent collisions, losing a fraction of $<20 \%$ of its kinetic energy on each collision.

Thus, instead of diffusion resulting from random steps instigated by collisions with the tiny fraction of incident hydrogen molecules having energy exceeding $0.23 \mathrm{eV}$ (the tiny fraction given by the Arrhenius $\exp \left(-U_{0} / \kappa T\right)$ factor), the random steps are now instigated by virtually all incident protons with each proton capable of producing multiple steps as it is trapped in the $-1 \mathrm{~V}$ potential well and so makes multiple collisions with water molecules. Although the protons are smaller in number than the hydrogen molecules by the ionization factor, the proton number is amplified by the OML focusing factor $T_{\mathrm{e}} / T_{\mathrm{i}} \sim 10^{2}$ for $T_{\mathrm{i}}=10^{-2} \mathrm{eV}$ and $V \simeq T_{\mathrm{e}} \approx 1 \mathrm{~V}$. On denoting the fractional ionization by $f$, since all incident protons produce random steps, the Arrhenius factor in Equation (23) would be nearly eliminated and the prefactor would be replaced by $f \mathrm{eV} / \kappa T_{\mathrm{i}} \mathrm{l}$, so for the nominal ionization fraction $f=10^{-6}$ of the Caltech experiment, an upper limit for the diffusion coefficient would become

$$
D^{*}=1.5 \times 10^{-2} \mathrm{~m}^{2} \mathrm{~s}^{-1},
$$




\section{Accreting molecule increases $b$}

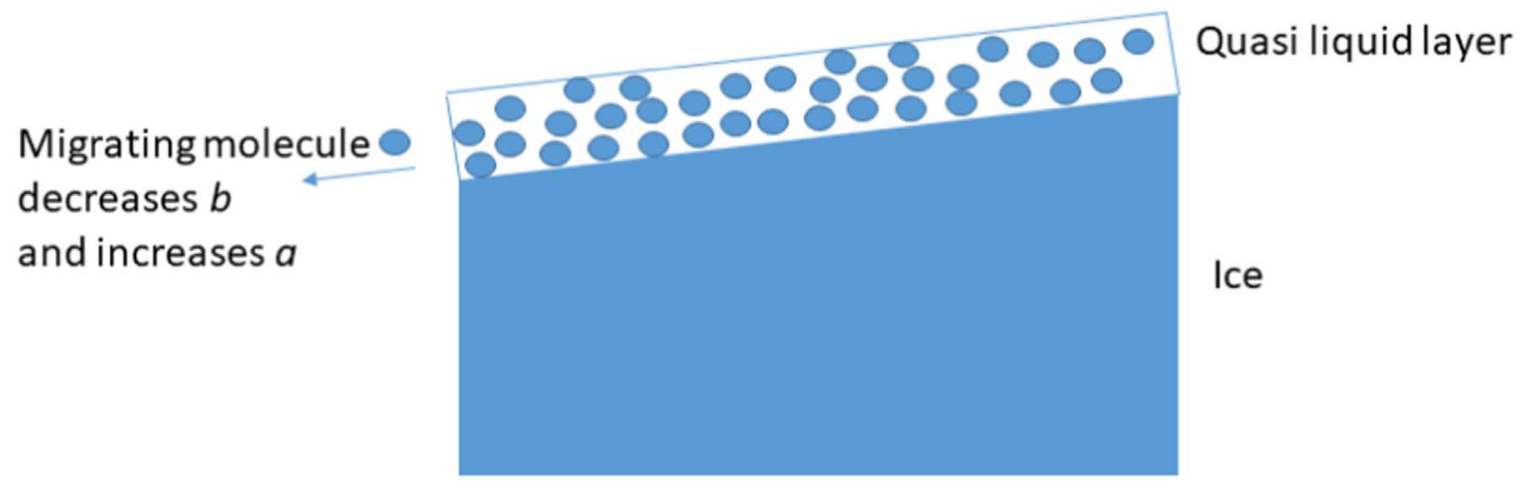

Figure 4. Zoomed-in view of surface showing quasi-liquid surface layer being replenished by accreting molecules coming from afar and being depleted by molecules migrating to the surface region having stronger normal electric field.

an increase by a factor of $10^{12}$ as compared to Equation (23). The mobility would be correspondingly higher and the time for a molecule to travel to the end correspondingly shorter. $D^{*}$ is an upper limit because it assumes that all incoming protons couple to adhered water molecules, and does not take into account that the incoming protons have a short lifetime because they recombine with incoming electrons so as to maintain constant charge on the ice grain surface. This tremendous increase in diffusivity of surface molecules would then easily explain the rapid growth of ice grains observed in the Caltech experiment. It also provides a mechanism for the astrophysical situation, because elimination of the Arrhenius $\exp \left(-U_{0} / \kappa T\right)$ factor enables the migration of molecules in the QLL to occur at arbitrarily low temperatures. In particular, it would enable migration at temperatures at $10 \mathrm{~K}$, whereas Equation (23) gives $D \sim 10^{-298}$ at such temperatures. This dependence of the aspect ratio on ion bombardment is consistent with the observation in Figure 3 of Chai \& Bellan (2015b) that $\alpha$ increases with increased fractional ionization as produced by applying more radio frequency power to the laboratory plasma (this increases plasma density and hence fractional ionization).

While this analysis is semiquantitative, it is expected that a more exacting analysis that accounts for geometric and collision details would retain this enormous increase. The main effect is the replacement of the Arrhenius factor $\exp (-60) \approx 10^{-26}$ by a much larger factor because incident ions (protons) have enough energy to detach one leg (the hydrogen atom) of a water molecule from the surface. According to this point of view, the probability of a molecule having energy between $U$ and $U+d U$ is $P(U) d U=$ $A \exp (-U / \kappa T) d U$, and so since $\int_{0}^{\infty} P(U) d U=1$, the norm is $A=1 / \kappa T$. The probability of having energy exceeding $U_{0}$ then is $\int_{U_{0}}^{\infty} P(U) d U=\exp \left(-U_{0} / \kappa T\right)$. Dislodging a leg of a water molecule attached to the surface requires $U_{0}=0.23 \mathrm{eV}$. Since the neutral gas and the surrounding water molecules have $\kappa T$ about $0.01 \mathrm{eV}$, if this dislodging is done by ambient gas molecules or surrounding water molecules, then $U_{0} / \kappa T$ is of the order of 25-60 depending on the details of the model. However, if the dislodging is done by ions that have fallen into the potential valley created by the electrons charging the dust grain, then $U_{0} / \kappa T$ is less than unity.
Other factors come into play such as the fractional ionization and the details of the collision process, but the importance of these pales into insignificance as compared to replacing a factor that is between $e^{-60}$ and $e^{-25}$ by a much larger factor. The negative potential of the ice grain likely reduces $U_{0}$ since Diallo et al. (2012) have shown that a surface electric field significantly increases diffusivity. Similarly, Ehre et al. (2010) have shown that the freezing temperature of a negatively charged water surface is lower than that of an uncharged surface, while the freezing temperature of a positively charged surface is higher. This suggests that negative charging reduces $U_{0}$ and positive charging increases $U_{0}$ since increased diffusivity corresponds to being more liquid-like.

Besides protons impacting the ice grain, there would also be small numbers of other ions such as $\mathrm{O}_{+}$impacting the grain. These might combine with protons to form water molecules and might also elastically collide with adhered water molecules. However, these effects would be in proportion to the abundance of oxygen relative to hydrogen and so would be much less important than the effect of protons impacting the ice grain. The enhancement of surface diffusion by ion bombardment is in many ways similar to the mechanism of plasma-enhanced chemical vapor deposition (e.g., see Hofmann et al. 2003).

Accretion combined with migration. We recall that $\dot{a}_{\text {accr }}$, $\dot{b}_{\text {accr }}$ are the rates of change of $a$ and $b$ from accretion, and define $\dot{a}_{\text {elong }}, \dot{b}_{\text {elong }}$ as the rates of change of $a$ and $b$ as a result of elongation due to surface migration from small $z$ to $z \sim a$; these effects are sketched in Figure 4. Because elongation conserves grain volume $\sim a b^{2}$, it is seen that

$$
\dot{a}_{\text {elong }}=-2 \alpha \dot{b}_{\text {elong }} \text {. }
$$

The values of $a$ and $b$ can change from both accretion and elongation, so

$$
\begin{aligned}
& \dot{a}=\dot{a}_{\text {accr }}+\dot{a}_{\text {elong }} \\
& \dot{b}=\dot{b}_{\text {accr }}+\dot{b}_{\text {elong. }} .
\end{aligned}
$$

Assume the cylindrical shell of mobile water molecules is a QLL with half-length $l_{b}$, where $l_{b}<a$. The thickness of this cylindrical shell is $\delta$. The number of QLL molecules moving radially inward in the length $l_{b}$ because of elongation is $2 \pi b l_{b} n \dot{b}_{\text {elong }}$, and the number of molecules moving axially outward from the shell is $2 \pi b \delta n v_{\tan }$, where $v_{\tan }$ is the tangential 
velocity of particles leaving the cylindrical shell as given by Equation (21). The number of radially inward molecules associated with the contraction of $b$ must equal the number of particles leaving the shell as a result of their axial motion, so

$$
\dot{b}_{\text {elong }}=-v_{\tan } \frac{\delta}{l_{b}}
$$

using Equation (28)

$$
\dot{a}_{\text {elong }}=2 \alpha v_{\tan } \frac{\delta}{l_{b}} .
$$

Thus

$$
\begin{gathered}
\dot{a}=\dot{a}_{\mathrm{accr}}+2 \alpha v_{\tan } \frac{\delta}{l_{b}} \\
\dot{b}=\dot{b}_{\mathrm{accr}}-v_{\tan } \frac{\delta}{l_{b}} .
\end{gathered}
$$

To have $b$ grow, it is necessary to have

$$
\dot{b}_{\text {accr }}>v_{\tan } \frac{\delta}{l_{b}}
$$

so molecules accrete on the cylindrical midsection faster than they are removed by surface migration to the ends.

The rate of increase of $\alpha$ is

$$
\begin{aligned}
\dot{\alpha} & =\frac{b \dot{a}-a \dot{b}}{b^{2}} \\
& =\frac{1}{b^{2}}\left(b\left(\dot{a}_{\mathrm{accr}}+2 \alpha v_{\tan } \frac{\delta}{l_{b}}\right)-a\left(\dot{b}_{\mathrm{accr}}-v_{\tan } \frac{\delta}{l_{b}}\right)\right) \\
& \simeq 3 v_{\tan } \frac{\delta}{b^{2}}
\end{aligned}
$$

where $l_{b} \simeq a=\alpha b$ is used.

Substitution of Equations (16), (17), and (21) into Equations (33) and (34) shows that $a, b$, and $\alpha$ evolve via the following three coupled equations:

$$
\begin{gathered}
\dot{a}=\frac{2 \alpha^{4 / 3} \sqrt{1-1 / \alpha^{2}}}{\ln \left(\frac{1+\sqrt{1-1 / \alpha^{2}}}{1-\sqrt{1-1 / \alpha^{2}}}\right)} \frac{n_{\mathrm{w}} m_{\mathrm{w}}}{\rho_{\mathrm{i}}} v_{\mathrm{w}}+2 \alpha p \frac{D}{\kappa T_{\mathrm{i}}} \frac{\alpha-1}{a} \frac{|V|}{b} \frac{\delta}{a} \\
\dot{b}=\frac{2 \alpha^{1 / 3} \sqrt{1-1 / \alpha^{2}}}{\ln \left(\frac{1+\sqrt{1-1 / \alpha^{2}}}{1-\sqrt{1-1 / \alpha^{2}}}\right)} \frac{n_{\mathrm{w}} m_{\mathrm{w}}}{\rho_{\mathrm{i}}} v_{\mathrm{w}}-p \frac{D}{\kappa T_{\mathrm{i}}} \frac{\alpha-1}{a} \frac{|V|}{b} \frac{\delta}{a} \\
\dot{\alpha}=3 p \frac{D}{\kappa T_{\mathrm{i}}} \frac{\alpha-1}{a} \frac{|V|}{b} \frac{\delta}{b^{2}}
\end{gathered}
$$

where $l_{b} \simeq a$ is used. These equations can be put in a dimensionless form by normalizing lengths to $b_{0}$, the initial value of $b$, and time to a reference time

$$
t_{\mathrm{ref}}=b_{0}\left(\frac{n_{\mathrm{w}} m_{\mathrm{w}}}{\rho_{\mathrm{i}}} v_{\mathrm{w}}\right)^{-1}
$$

which is the time for a sphere of initial radius $b_{0}$ to double its radius via accretion only. Normalized quantities are indicated by a bar and normalized equations are obtained by multiplying Equations (37) and (38) by $t_{\text {ref }} / b_{0}$ and Equation (39) by $t_{\text {ref. }}$.
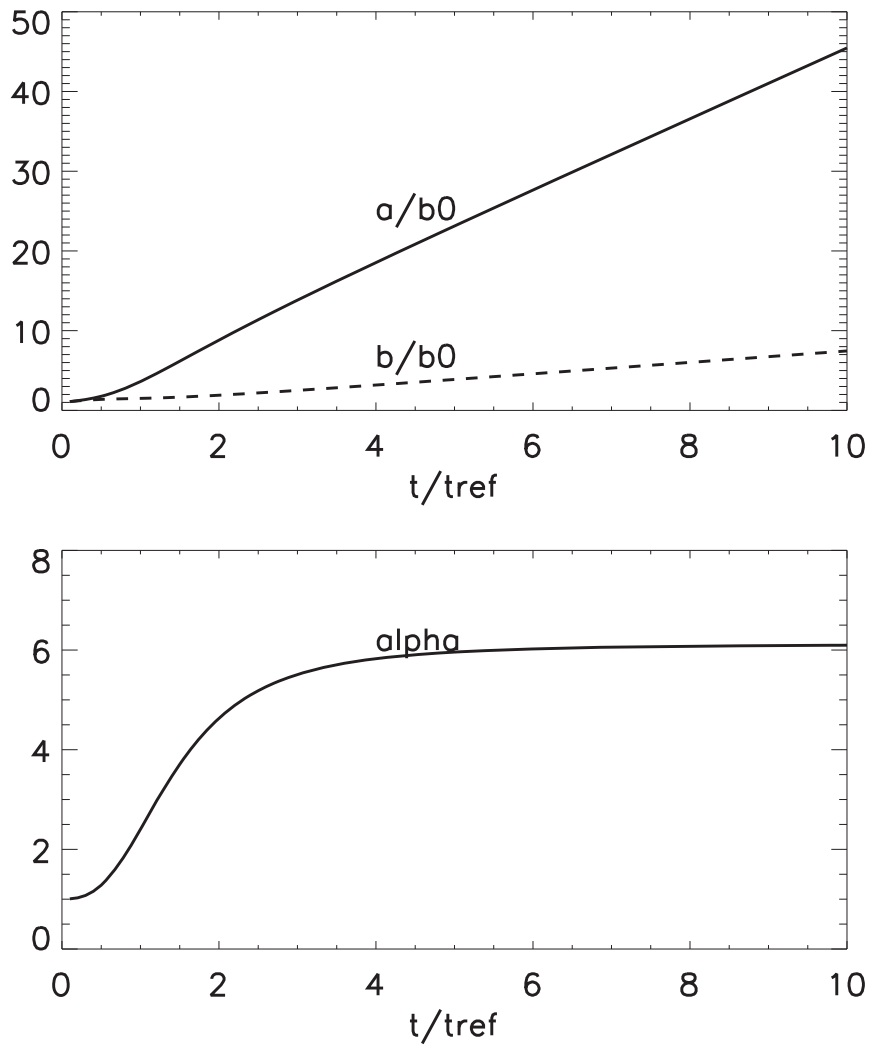

Figure 5. Numerical solution of Equations (41)-(43) for initial $\alpha=1.001$ and $\lambda=8.00905$

The normalized equations are

$$
\begin{gathered}
\frac{d \bar{a}}{d \bar{t}}=\frac{2 \alpha^{4 / 3} \sqrt{1-1 / \alpha^{2}}}{\ln \left(\frac{1+\sqrt{1-1 / \alpha^{2}}}{1-\sqrt{1-1 / \alpha^{2}}}\right)}+2\left(1-\frac{1}{\alpha}\right) \frac{\lambda}{\bar{b}^{3}} \\
\frac{d \bar{b}}{d \bar{t}}=\frac{2 \alpha^{1 / 3} \sqrt{1-1 / \alpha^{2}}}{\ln \left(\frac{1+\sqrt{1-1 / \alpha^{2}}}{1-\sqrt{1-1 / \alpha^{2}}}\right)}-\frac{1}{\alpha}\left(1-\frac{1}{\alpha}\right) \frac{\lambda}{\bar{b}^{3}} \\
\frac{d \alpha}{d \bar{t}}=3\left(1-\frac{1}{\alpha}\right) \frac{\lambda}{\bar{b}^{4}}
\end{gathered}
$$

where

$$
\lambda=\frac{p|V|}{\kappa T_{\mathrm{i}} b_{0}^{3}} \frac{\rho_{\mathrm{i}}}{n_{\mathrm{w}} m_{\mathrm{w}}} \frac{D \delta}{v_{\mathrm{w}}} .
$$

Figure 5 shows a numerical solution of Equations (41)-(43). The terms containing logarithms in Equations (41) and (42) are of order unity for $1<\alpha<5$. Varying parameters in repeated numerical solutions of these equations show that when $\lambda \approx 1+\ln \left(\frac{1}{\alpha_{0}-1}\right)$, where $\alpha_{0}$ is the initial value of $\alpha, \alpha$ grows exponentially in a few reference times and then saturates at a value that depends sensitively on the precise value of $\lambda$. Since $\lambda$ depends on $D, \delta$, and $b_{0}$, all of which are poorly known, the value of $\lambda$ can only be estimated. Using $b_{0}=10 \mathrm{~nm}, \delta=1 \mathrm{~nm}$, and $D=3 \times 10^{-14}$ as predicted by Equation (23) for $T_{\mathrm{i}}=190 \mathrm{~K}$ gives $\lambda=6 \times 10^{-2}$, which is too small to give growth in $\alpha$. However, an increase of the value of $D$ to $D=4.4 \times 10^{-12}$ based on the presumption that proton bombardment increases diffusion gives $\lambda=8$, which results in $\alpha$ saturating at $\alpha=5$ when $\alpha_{0}=1.001$ (as shown in Figure 5). After saturation of $\alpha$, 
the radii $a$ and $b$ continue to grow linearly in time. This saturation of $\alpha$ can be seen from the terms containing $\lambda$ becoming unimportant in Equations (41)-(43) when $\bar{b}^{3} \gg \lambda$. For the Caltech experiment $t_{\text {ref }}=8 \mathrm{~ms}$, so the prediction that $\alpha$ is constant for times much greater than $8 \mathrm{~ms}$ is consistent with Figure 4(b) in Marshall \& Bellan (2020), which shows the aspect ratio remaining constant at $\alpha \approx 4.5$ when $a$ increases from 20 to $80 \mu \mathrm{m}$ in a $10 \mathrm{~s}$ time interval.

While this model identifies the transition from early times, when $\alpha$ increases rapidly, to later times, when $\alpha$ and $b$ increase at constant $\alpha$, it does not identify the size saturation that was observed in the experiment after tens of seconds. Possible explanations for the size saturation are that the gas-phase water molecules have been depleted by the large surface area of large ice grains or that the ice grains start to interfere with one another so that instead of presuming that incident water molecules come from infinity, one has to consider that they are only coming from the vicinity of the next ice grain. This model should be considered a "toy" model that, while capturing some of the essential mechanisms, also leaves out many important effects. In particular, Equation (21) provides only an order of magnitude estimate of the migration velocity because of the simplified estimate for $\nabla E$. The model seems overly sensitive to the choice of $D$, which suggests that there should be some negative feedback mechanism that is not being taken into account.

Furthermore, the inverse dependence of $\lambda$ on $n_{\mathrm{w}}$ is contrary to the observations in Chai \& Bellan (2015b), where it was seen that $\alpha$ increases with $n_{\mathrm{w}}$. Examination of Equations (37) and (38) shows that the inverse dependence of $\lambda$ on $n_{\mathrm{w}}$ results from the first (i.e., accretion) term in these respective equations containing a factor $n_{\mathrm{w}}$ but from the second term (i.e., elongation) not containing $n_{\mathrm{w}}$. In order to make $\lambda$ proportional to $n_{\mathrm{w}}$ it would be necessary for the elongation terms to have a greater than linear dependence on $n_{\mathrm{w}}$. This could happen if surface diffusion depended on $n_{\mathrm{w}}$ such that gas-phase water molecules collided with loosely bound surface molecules and enhanced diffusion. Measurements of surface diffusivity have generally been done at atmospheric pressure with no plasma, so there is no information on how gas-phase density or plasma affects surface diffusion. The need for $D$ to depend on $n_{\mathrm{w}}$ is very evident if one considers astrophysics situations where $n_{\mathrm{w}}$ is orders of magnitude smaller than that in the laboratory. If $D$ did not depend on $n_{\mathrm{w}}$, then the first terms on the right-hand side of Equations (37) and (38) would become negligible as $n_{\mathrm{w}}$ became small, whereas the second terms would remain unchanged. A possible means by which $D$ could depend on $n_{\mathrm{w}}$ is that the presence of plasma would dissociate gas-phase water molecules to produce hydrogen and oxygen atoms and then some of the oxygen atoms would be ionized by the plasma. These oxygen ions would fall into the $-1 \mathrm{~V}$ potential well of the negatively charged dust grain and collide with loosely bound water molecules adhering to the surface. These collisions would enhance the diffusion of the loosely bound surface molecules and so $D$ would depend on $n_{\mathrm{w}}$.

Despite these shortcomings, the model does identify an instability showing that very small grains deviating slightly from pure spherical symmetry will quickly become prolate ellipsoids and that the aspect ratio will be conserved as the grains grow to large size. It also indicates the need for diffusion enhancement via proton bombardment and the need to consider whether surface diffusivity is dependent on gas-phase water vapor density.

Excessiveness of surface tension arguments. The decrease of electrostatic energy at constant charge $Q^{2} / 2 C$ and the increase of surface potential energy $\Sigma S$, where $\Sigma$ is the surface tension, suggest that elongation might occur as a result of the selfelectrostatic force of the ellipsoid as has been discussed in the context of liquid water drops by Shchukin \& Grigorev (1998) and by Holgate \& Coppins (2016). According to this point of view, the mutual repulsion between the charges localized at the two ends would stretch the ice grain until an equilibrium is reached with the opposing force provided by surface tension. Thus an equilibrium would develop when the sum of electrostatic and surface tension potential energies is at a minimum, i.e., when

$$
U=\Sigma S+Q^{2} / 2 C
$$

is at a minimum. This approach explains the behavior of liquid drops. However, it fails for the QLL on ice grains if the conventional value of water surface tension $\Sigma \simeq 0.1 \mathrm{~N} \mathrm{~m}^{-1}$ is used because $\Sigma S$ becomes orders of magnitude larger than $Q^{2} / 2 C$ for ice grains in the size range of nanometers to hundreds of microns. This is demonstrated in the situation where the grain is spherical, so $S=4 \pi R^{2}$ and $C=4 \pi \varepsilon_{0} R$; thus

$$
U=4 \pi R^{2} \Sigma+\frac{Q^{2}}{8 \pi \varepsilon_{0} R}
$$

which has a minimum at

$$
R=\frac{1}{4}\left(\frac{Q^{2}}{\pi^{2} \varepsilon_{0} \Sigma}\right)^{1 / 3} .
$$

This minimum can be expressed in terms of the charge voltage using $Q=C V$, so $R=\varepsilon_{0} V^{2} / 4 \Sigma \simeq 10^{-11} \mathrm{~m}$ for $V=1 \mathrm{~V}$. Clearly, the surface tension would have to be reduced by many orders of magnitude for the ice grains observed in the Caltech experiments to be explained in terms of a competition between electrostatic forces and surface tension in the QLL coating the ice. This suggests that the effective surface tension in the QLL is extremely low, while the ice itself has no surface tension. The easy migration of surface water molecules is not consistent with the concept of surface tension since surface tension requires a stretched chain of molecules wrapped around the periphery. Furthermore, the water molecules in the QLL have their dipole moments pointed toward the charged surface (see Figure 1 of Murphy 1953), so they do not attract one another via their dipole moments. Thus, they do not form a stretched chain wrapped around the periphery since such a chain requires the molecules to have their dipole moments tangential to the surface.

The necessity for the QLL to have very small surface tension can also be seen by making a local analysis at the end $z=a$, which is the region of the smallest radius of curvature, in a manner similar to that used for Taylor cones (Taylor 1964). The stress from surface tension is $\Sigma / r_{\mathrm{c}}$, where $r_{\mathrm{c}}$ is the local radius of curvature, and this provides an inward force. The stress from the electric field is $\varepsilon_{0} E^{2} / 2$, where $E=V / r_{\mathrm{c}}$, and this provides an outward force. The electric stress will thus overwhelm the surface tension if $r_{\mathrm{c}}<\varepsilon_{0} V^{2} / 2 \Sigma$. Using water surface tension $\Sigma=0.1 \mathrm{~N} \mathrm{~m}^{-1}$ and assuming $V=T_{\mathrm{e}}$ with $T_{\mathrm{e}}=1 \mathrm{eV}$ gives $r_{\mathrm{c}}<0.04 \mathrm{~nm}$, but a sphere having such a 
small radius would not have any electrons since the radius for a sphere with one electron and charged to $1 \mathrm{~V}$ is $1.4 \mathrm{~nm}$. It should be noted that Taylor cones involve potentials of $10^{4} \mathrm{~V}$ corresponding to $r_{\mathrm{c}}$ of a few millimeters.

Gladich et al. (2011) show a molecular dynamics simulation of an ice crystal and its QLL in their Figure 2. This figure and the analysis provided by Gladich et al. (2011) show that the QLL becomes thinner at lower temperatures and at $230 \mathrm{~K}$, the lowest temperature in the simulation, there are gaps in the QLL which has a thickness less than two molecular layers. Such gaps would eliminate surface tension since surface tension results from attraction between molecules in the QLL-there cannot be any attraction between molecules if there are no molecules. This supports the contention that at very low temperatures surface tension ceases to be operative and that molecular diffusion dominates.

Prolate versus oblate. Most of the discussion here could also be used to argue for a tendency to form oblate ice grains (i.e., $\alpha \ll 1)$ since the electric field on the rim of an oblate grain is stronger than that on a pancake-like flat surface by the factor $\alpha^{-1}$. However, the experiments showed no evidence of the formation of oblate structures, suggesting there is some underlying difference between oblate and prolate. One possible explanation would be that the progression from having no electrons on the ice surface to having a large number necessarily involves a stage where there are just two electrons on the surface. At this stage, the ice dimensions would be $d=2 q_{\mathrm{e}} / 4 \pi \varepsilon_{0} V \simeq 3 \mathrm{~nm}$, but this is the dimension of the QLL so it is likely that a $3 \mathrm{~nm}$ grain does not consist of fully formed ice and instead is rather pliable. The repulsive force between the two electrons would thus create a prolate shape from this pliable structure, and subsequent growth would maintain this prolate shape. The most primitive oblate shape requires defining a plane rather than a line. A plane would require three electrons, but this would happen later and so be preempted by the prolate shape requiring only two electrons.

Situation of positively charged grains. It has thus far been assumed that dust grains are negatively charged. However, in the presence of ultraviolet (UV) light with photon energy exceeding the work function of the dust material, dust grains emit photoelectrons. If the UV intensity is sufficiently strong, this process will dominate and result in the dust grains becoming positively charged (Watson 1972; Spitzer 1998). In this situation photoemitted electrons leave the grain while, at the same time, electrons from the background plasma arrive on the grain. Because ion velocities are slow compared to electron velocities, the ion flux from the background plasma can be neglected compared to this two-way electron traffic. An equilibrium dust charge then results when the flux of photoemitted electrons leaving the dust grain balances the flux of plasma electrons arriving on the dust grain.

In the previously considered situation of negatively charged dust grains the dust potential is independent of plasma density because the competing charging processes of inward ion flux and inward electron flux are both proportional to plasma density. In contrast, for positively charged grains the inward flux of plasma electrons depends on the background plasma density, whereas the photon flux does not. Thus, for positively charged dust grains the dust potential will become more positive as the background plasma density decreases. However, the magnitude of this positive potential has an upper bound that is given by the difference between the UV photon energy and the work function. This upper bound is due to the fact that photoemitted electrons cannot escape the dust grain if its positive potential exceeds the initial electron kinetic energy as the positive potential acts as a well from which the electrons must escape. The initial kinetic energy of photoemitted electrons corresponds to the difference between the photon energy and the work function, and this difference is at most a few electronvolts for photons having significant flux. This means that, as is the case for negatively charged grains, the magnitude of the potential of positively charged grains will be between one and a few electronvolts.

Photoemission depends on the work function, the photon absorption efficiency, and the electron yield per absorbed photon. As discussed in Abbas et al. (2006), the work function depends on the grain size, the surface condition, and the type of material. Photoemission occurs only when the photon energy exceeds the work function, which is $4-8 \mathrm{eV}$ for typical grain materials (Kimura 2016). If $R$ represents a nominal grain radius and $\lambda$ is the wavelength of the UV light, photon absorption efficiency scales as $2 \pi R / \lambda$ if $2 \pi R \ll \lambda$. However, if $2 \pi R \gg \lambda$, then the photon absorption efficiency is of order unity. There is controversy regarding the photon yield with values predicted to range from $10^{-3}-10^{-2}$ to being of order unity (Abbas et al. 2006; Kimura 2016).

If it is assumed that UV radiation is sufficiently intense to make ice grains positive, then the chain of events would differ in significant ways from the previous discussion of negative grains. First of all, if nucleation is homogeneous, then it would require starting with the photoionization of a water molecule (Suwannakham et al. 2018). Because the dipole attractive force does not depend on the sign, a positively charged water molecule would attract neutral water molecules as is the case for negative charging; the only difference would be in the orientation of the attracted water molecule. The orientation of a just-arrived water molecule clinging to the ice grain surface would be inverted as compared to the situation of a negatively charged ice grain. Specifically, Figure 3 would have to be changed to have the electric field (black arrows) pointing away from the ice grain, and the water molecule's oxygen atom would contact the ice grain surface rather than the molecule's hydrogen atoms. This is because in a water molecule the oxygen atom is slightly negative while the hydrogen atoms are slightly positive. Thus, for a water molecule in loose contact with a positively charged grain, there is only one point of contact (the oxygen atom) rather than two points as is the case for a negatively charged grain (the two hydrogen atoms). This means that the bipedal model in Murphy (1953) will no longer apply, so a water molecule will either be attached or not. Figure 9 of Dezfoli et al. (2013) shows a molecular dynamics simulation of water molecules interacting with positively and negatively charged carbon nanotubes and reveals an inversion of the water molecule orientation when the polarity of the nanotube charge is reversed; this inversion supports the argument made here.

A positively charged grain will not attract protons and so, in contrast to negatively charged grains, there will not be an inward flux of protons. These protons were predicted to be energetic as a result of falling into the negative potential of the dust grain and so they would enhance the surface diffusion of loosely attached water molecules. However, for a positively charged grain this effect of energetic protons enhancing diffusion would be replaced by a similar effect resulting from negative ions falling into the potential well associated with the 
positive charge. These negative ions would result from electrons in the background plasma becoming attached to neutral gas atoms (Millar et al. 2017).

Once the ice grain has grown sufficiently large that $p E /$ $\kappa T \ll 1$, where $E \sim V / R$ with $V$ being the potential of a few volts and $R$ being the nominal size, there will no longer be surface diffusion. As in the case of negative grains, the grain could then grow in a self-similar fashion because of the geometrical effect of the ends of a prolate ellipsoid subtending a larger solid angle than the sides; this is the constant $\alpha$ limit in the bottom plot in Figure 5.

The above discussion qualitatively outlines how the situation would change if the grains are positive; the existence of changes is consistent with the observation by Ehre et al. (2010) that positively charged ice behaves differently from negatively charged ice. The uncertainties in this outline suggest that laboratory experiments applying UV radiation to cooled water vapor would be worthwhile. The goal of such experiments would be to determine whether in the presence of UV but in the absence of plasma, cooled water vapor spontaneously nucleates to form growing, positively charged ice grains. If UVgenerated, homogeneously nucleated ice grains indeed exist in astrophysical situations, it should be possible to replicate this generation in the laboratory since the presumed intensity and wavelength of the UV radiation, the water vapor density, and the background gas parameters are not extreme.

By starting with solid grains in cooled water vapor and no plasma, it would also be possible to determine whether heterogeneous nucleation and ice growth occur in the presence of UV but without plasma. Because starting with a solid core defines the initial morphology, this type of starting precludes the surface diffusion at small scales that resulted in a prolate shape. It is consequently expected that heterogeneous, UVmediated nucleation will result in a grain that is in general not prolate.

\section{Summary}

The Caltech experiment showed that spontaneously nucleating water ice grains in a weakly ionized plasma tend to be prolate. The observed values of the aspect ratio $\alpha$ depended on many experimental factors with largest $\alpha$ obtained at low pressure with the lightest background gas (hydrogen). An $\alpha \sim 20$ was obtained with methanol ice in a $10 \mathrm{mT}$ hydrogen background, and nearly as large $\alpha$ was obtained with water ice (see Figures 8(b) and 2(a) of Chai \& Bellan 2015a). Dusty plasma analysis shows that the ice grain surface potential is fixed to be approximately the temperature of the electrons in the surrounding plasma. This means that an ice grain with dimensions of the order of tens of nanometers can have a dipole energy $p E$ comparable to the thermal energy $\kappa T_{\mathrm{w}}$ of water molecules in the QLL coating the ice grain. This would cause the extremely small ice grain to become a prolate ellipsoid because molecules on the surface would migrate to the region of strongest $E$, which is at the ends of the prolate ellipsoid. The aspect ratio would be maintained or even grow when the dust grain becomes larger. This dipole energy effect ceases to work when the grain size exceeds a few tens of nanometers.

The surface diffusivity of surface water molecules calculated with no plasma effects appears to be too small to provide the surface migration toward regions of strong electric field required to explain an ice grain tending to be prolate. This inadequacy becomes extreme at lower temperatures. However, when the plasma effect of ice grain bombardment by energetic ions is taken into account, the diffusivity of surface water molecules increases by as much as 12 orders of magnitude, so now there is no problem invoking migration of surface molecules to regions of strong normal electric fields. Thus, a two-stage scenario results where at sizes of the order of tens of nanometers, the ice grains become prolate ellipsoids and then this profile is maintained by the prolate ellipsoid ends subtending a much larger solid angle than the sides as the ice grain grows to much larger sizes.

These models require surface tension to be negligible compared to that of ordinary water as the energy associated with normal surface tension would overwhelm the energy available to drive the surface migration of water molecules. This lack of surface tension is consistent with the existence of a very thin quasi-liquid surface layer where the molecules are mainly attached to the ice surface rather than to one another.

Since these phenomena can maintain and even increase the aspect ratio $\alpha$ and since the experimental observations (Chai \& Bellan 2013, 2015a, 2015b; Marshall et al. 2017) indicate the spontaneous formation of prolate ellipsoids in a wide variety of conditions, there is good reason to conclude that astrophysical ice grains tend to be prolate ellipsoids and not spheres. This provides the prolate ellipsoidal grains required by models of grain alignment and indicates that astrophysical ice grain growth differs from the spherical growth predicted by models that do not take charging and associated dusty plasma effects into account. As demonstrated by the formation of prolate methanol and acetone ice grains by Chai \& Bellan (2015a), these effects should also occur with non-water polar molecules.

The scenario may change if UV radiation is so intense that ice grains become positively charged. In particular, the details of how a water molecule initially attaches to an ice grain will differ and enhanced surface diffusion by bombardment of energetic positive ions would have to be replaced by bombardment by negative ions.

Supported by the National Science Foundation/Department of Energy Partnership in Basic Plasma Science and Engineering via USDOE Award DE-SC0020079.

\section{ORCID iDs}

\section{P. M. Bellan (iD https://orcid.org/0000-0002-0886-8782}

\section{References}

Abbas, M. M., Tankosic, D., Craven, P. D., et al. 2006, ApJ, 645, 324 Acharyya, K., Hassel, G. E., \& Herbst, E. 2011, ApJ, 732, 73

Allen, J. E. 1992, PhyS, 45, 497

Barkan, A., D’Angelo, N., \& Merlino, R. L. 1994, PhRvL, 73, 3093

Bartlett, J. T., Heuval, A. P., \& Mason, B. J. 1963, ZaMP, 14, 599

Chai, K.-B., \& Bellan, P. M. 2013, GeoRL, 40, 6258

Chai, K.-B., \& Bellan, P. M. 2015a, ApJ, 802, 112

Chai, K.-B., \& Bellan, P. M. 2015b, JASTP, 127, 83

Davis, L., Jr., \& Greenstein, J. L. 1951, ApJ, 114, 206

Dezfoli, A. R. A., Mehrabian, M. A., \& Rafsanjani, H. H. 2013, Korean J Chem. Eng., 30, 693

Diallo, S. O., Mamontov, E., Nobuo, W., Inagaki, S., \& Fukushima, Y. 2012, PhRvE, 86, 021506

Doppenschmidt, A., \& Butt, H.-J. 2000, Langm, 16, 6709

Ehre, D., Lavert, E., Lahav, M., \& Lubomirsky, I. 2010, Sci, 327, 672

Gladich, I., Pfalzgraff, W., Marsalek, O., et al. 2011, PCCP, 13, 19960

Gold, T. 1952, MNRAS, 112, 215

Hall, J. S. 1949, Sci, 109, 166

Hiltner, W. A. 1949, Sci, 109, 165 
Hofmann, S., Ducati, C., Robertson, J., \& Kleinsorge, B. 2003, ApPhL, 83,135

Holgate, J. T., \& Coppins, M. 2016, PhRvE, 93, 033208

Jones, R. V., \& Spitzer, L., Jr. 1967, ApJ, 147, 943

Kimura, H. 2016, MNRAS, 459, 2751

Lazarian, A. 2007, JQSRT, 106, 225

Libbrecht, K. G., \& Tanusheva, V. M. 1999, PhRvE, 59, 3253

Marshall, R. S., \& Bellan, P. M. 2020, RScI, 91, 063504

Marshall, R. S., Chai, K.-B., \& Bellan, P. M. 2017, ApJ, 837, 56

Martin, P. G. 1971, MNRAS, 153, 279

Mason, B. J., Bryant, G. W., \& Van den Heuvel, A. P. 1963, PMag, 8, 505

Mathis, J. S., Rumpl, W., \& Nordsieck, K. H. 1977, ApJ, 217, 425

Millar, T. J., Walsh, C., \& Field, T. A. 2017, ChRv, 117, 1765

Murphy, E. J. 1953, JChPh, 21, 1831
Price, W. S., Ide, H., \& Arata, Y. 1999, JPCA, 103, 448

Shchukin, S. I., \& Grigorev, A. I. 1998, Technical Physics, 43, 1314

Shimizu, S., Klumov, B., Shimizu, T., et al. 2010, JGR, 115, D18205

Shukla, P., \& Mamun, A. 2002, Introduction to Dusty Plasma Physics (Bristol: IOP Publishing)

Spitzer, L. 1998, Physical Processes in the Interstellar Medium (1st ed.; New York: Wiley)

Spitzer, L., Jr. 1941, ApJ, 93, 369

Suwannakham, P., Chaiwongwattana, S., \& Sagarik, K. 2018, RSCAd, 8, 36731

Taylor, G. 1964, RSPSA, 280, 383

Watson, W. D. 1972, ApJ, 176, 103

Zwillinger, D. (ed.) 2011, CRC Standard Mathematical Tables and Formulae (Boca Raton, FL: CRC Press) 